\title{
Cube Arithmetic: Improving Euler Method for Ordinary Differential Equation Using Cube Mean
}

\author{
Nooraida Samsudin, Nurhafizah Moziyana Mohd Yusop, Syahrul Fahmy, \\ Anis Shahida Niza binti Mokhtar \\ TATI University College, Teluk Kalong, Kemaman, Terengganu, \\ Universiti Pertahanan Nasional Malaysia, Kem Sungai Besi, Kuala Lumpur, Malaysia
}

\begin{tabular}{|c|c|}
\hline Article Info & ABSTRACT \\
\hline Article history: & \multirow{8}{*}{$\begin{array}{l}\text { The Euler method is a first-order numerical procedure for solving Ordinary } \\
\text { Differential Equation (ODEs) problems. It is an effective and easy method to } \\
\text { solve initial value problems. Although Euler provides simple procedure for } \\
\text { solving ODEs, there have been issues such as complexity, time of processing } \\
\text { and accuracy that compelled the use of other, more complex, methods. } \\
\text { Improvements to the Euler method have attracted much attention resulting in } \\
\text { numerous modified Euler methods. This paper proposes Cube Arithmetic, a } \\
\text { modified Euler method with improved accuracy. The efficiency of Cube } \\
\text { Arithmetic was compared with Euler Arithmetic and tested using SCILAB } \\
\text { against exact solutions. Results indicate that not only Cube Arithmetic } \\
\text { provided solutions that are similar to exact solutions at small step size, but } \\
\text { also at higher step size, hence producing more accurate results. }\end{array}$} \\
\hline Received May1, 2018 & \\
\hline Revised Jun 2, 2018 & \\
\hline Accepted Jun 11, 2018 & \\
\hline Keywords: & \\
\hline Cube arithmetic & \\
\hline Euler & \\
\hline SCILAB & \\
\hline
\end{tabular}

Copyright (C) 2018 Institute of Advanced Engineering and Science. All rights reserved.

Corresponding Author:

Nooraida Samsudin,

TATI University College, Teluk Kalong, Kemaman, Terengganu,

Universiti Pertahanan Nasional Malaysia, Kem Sungai Besi, Kuala Lumpur, Malaysia.

Email: nooraida@tatiuc.edu.my

\section{INTRODUCTION}

Numerical method is widely used in computer science. It helps researchers, engineers, and students to approximate the solution of complicated problems so that the final solution consists of only addition, subtraction and multiplication operations. Numerical method forms an important part of solving Initial Value Problems (IVPs) in Ordinary Differential Equations (ODEs), especially in cases where the exact solution is difficult to obtain or in the absence of closed form analytic formula.

The Euler method is a first-order numerical procedure for solving ODEs with a given initial value. It is an effective [1] and the easiest method [2] to solve IVPs in ODEs. Euler method has been use in variety field such as in communication and security to get better solution. Regarding [3], Euler method has better performance in modeling uncertainties. It is suppeoted by [4] where as experimental results indicate that it is much simpler and faster, more sensitive and robust. Although Euler provides simple procedure for solving ODEs, there are issues that have compelled the use of other, more complex, methods such as Runge Kutte. One of them is "accuracy" as reported by [5-6]. Improvements to the Euler method have attracted much attention including [1-2], [5-7], resulting in modified Euler methods.

This paper is aim to discover a new algorithm as accurate as possible with exact solution by using average concept. The new algorithm proposed in this paper name it as Cube Arithmetic. This idea to develop this new algorithm is by enhance other algorithm proposed by Qureshi [7] and Nurhafizah [8]. Qureshi used average of arithmetic mean for two points at coordinate $y=[f(x n, y n)+f(x n+1, y n+1)] / 2$. The equation is referring as Euler Arithmetic shows improvement in accuracy and speed compared to Euler Method. [8] uses concept of Qureshi [7] but choose Harmonic mean and Contraharmonic mean in the equation . 
Thus, this paper proposes a modified Euler method using the Cube mean concept. The method was constructed by extracting the Euler Arithmetic (EA) method in [9] and using Cube mean in the equation. Propose algorithm is compared between modified Euler known as Euler Arithmetic with the exact solution. The accuracy of the proposed method is demonstrated by the comparing error results of with step sizes of $h$ $0.1,0.01$ and 0.001 .

\section{PROPOSED ALGORITHM}

The algorithm is based on the Cube Mean in Euler Arithmetic. Euler Arithmetic (EA) is a wellknown technique for improving the Euler method. Euler methods used in [1] and [2] were extracted as basis for the algorithm. Cube mean within two coordinate points of function were used to improve the methods. Equation (1) is a basic formula of the Euler method.

$$
y_{\mathrm{n}+1}=y_{n}+\Delta t f\left(t_{0}, y_{0}\right)
$$

Equation (2) by [1] was modified using the average concept.

$$
y_{n+1}=y_{n}+h f\left(x_{n}+\frac{h}{2}, y_{n}+\frac{h}{2}\left(\frac{f\left(x_{n,} y_{n}\right)+f\left(x_{n}+h\right),\left(y_{n}+h f\left(x_{n}, y_{n}\right)\right.}{2}\right)\right.
$$

The proposed average in cube mean for the two midpoints is written as Equation (3)

$$
y_{n+1}=y_{n}+h f\left(x_{n}+\frac{h}{2}, y_{n}+\frac{h}{2} \sqrt[3]{\left(\frac{f\left(x_{n,} y_{n}\right)^{3}+\left(f\left(x_{n}+h\right),\left(y_{n}+h f\left(x_{n}, y_{n}\right)\right)^{3}\right.}{2}\right)}\right.
$$

Modifications to the slope of the function at estimated midpoints of $\left(x_{0}, y_{0}\right)$ and $\left(x_{1}, y_{1}\right)$ would improve the stability and accuracy of Euler.

\section{RESEARCH METHOD}

There are a few processes to present a mathematical model. First, it can be presented by mathematical software and algorithm development. Before using the mathematical software, algorithm development must be done first. Algorithm refers as instructions for solving problem using simple language [10-11]. According to [12], construction process in mathematical software includes as follows.

a) The design and analysis algorithms

b) Algorithm coding

c) Testing

d) Details documentation

e) Distribution and maintenance of the software

Algorithm that has been developed will be converting to the programming language. In this study, the code program is written in SCILAB 6.0 to test the effectiveness of the algorithm. The effectiveness of the algorithm will be identifying by taking itaccuracy and time of taken to process the algorithm. This paper will focus on accuracy by comparing the exact solution and solution between proposed method known as Cube Euler Arithmetic and modified Euler naming as Arithmetic Euler.

Cube Arithmetic (CA), the proposed algorithm in Equation (3), was represented using SCILAB 6.0. SCILAB is a powerful, low-cost [13]-[14] and flexible tool for researchers, engineers, and students to use in mathematical computer application.Scilab was developed by the French National Institute for Informatics and Automation Research (INRIA) [15].The effectiveness of CA was determined through the comparis on of both CA and modified EA solutions, focusing on accuracy and processing time.

\section{RESULTS AND ANALYSIS}

Results of three first order ODEs were recorded. These results were obtained by testing CA with three sets of linear ODEs using three different step sizes: 0.1, 0.01 and 0.001 . These sets were tested for efficiency using the minimum and maximum error for the whole cycle. Table 1 illustrates the exact solutions 
for the ODEs. Table 2 illustrates the comparison results between EA and CA methods. Relative error based on [17] was calculated as:

$$
\text { Error= }[E x-E v], E x=\text { Exact_value and } E v=E u l e r \text { 's_modified_value }
$$

Table 1. Set of Problem First Order ODEs

\begin{tabular}{ccccc}
\hline Equation & Exact Solution & Initial Values & Interval of Integration & Source \\
\hline $\boldsymbol{y}^{\prime}=\mathbf{- 0 . 5 y}$ & $\mathrm{e}^{(-1.0 .5 x)}$ & $y(0)=0$ & $0 \leq x \leq 20$ & {$[16]$} \\
$\mathbf{y}^{\prime}=-\mathbf{y}$ & $\mathrm{e}^{(-x)}$ & $y(0)=1$ & $0 \leq x \leq 20$ & {$[16]$} \\
$\mathbf{y}^{\prime}=\mathbf{- 1 0 y}$ & $\mathrm{e}^{(-10 x)}$ & $y(0)=1$ & $0 \leq x \leq 20$ & {$[16]$} \\
\hline
\end{tabular}

Table 2. Results of Errors Using Various Step Size $h$

\begin{tabular}{ccccccc}
\hline Method & \multicolumn{3}{c}{ Euler Arithmetic } & \multicolumn{3}{c}{ Cube Arithmetic } \\
\cline { 2 - 7 } Step Size & $\mathrm{h}=0.001$ & $\mathrm{~h}=0.01$ & $\mathrm{~h}=0.1$ & $\mathrm{~h}=0.001$ & $\mathrm{~h}=0.01$ & $\mathrm{~h}=0.1$ \\
\hline Problem 1 & 0 & 0 & 0.000083 & 0 & 0.000001 & 0.000076 \\
Problem 2 & 0 & 0.000003 & 0.000355 & 0 & 0.000003 & 0.00302 \\
Problem 3 & 0 & 0.000401 & 0.117900 & 0 & 0.000300 & 0.029020 \\
\hline
\end{tabular}

The results for each method were compared to the exact solution using maximum error. It is evident from Table 2 that the proposed CA method provided a more accurate result than EA at higher $h$ sizes. Smaller step size produced similar results for both methods.

Problem 1 demonstrated that the results of the CA method is better than EA at $h=0.1$ with a maximum error of 0.000076 and 0.000083 respectively. Figure 1 illustrates the differences between the two methods. Results at $h=0.01$ showed that CA scored 0.000001 compared to EA at 0 . Both methods scored the same at $h=0.001$. Problem 2 demonstrated that the results for both CA and EA methods are almost the same at $h=0.1,0.01$ and 0.001, as shown in Figure 2.

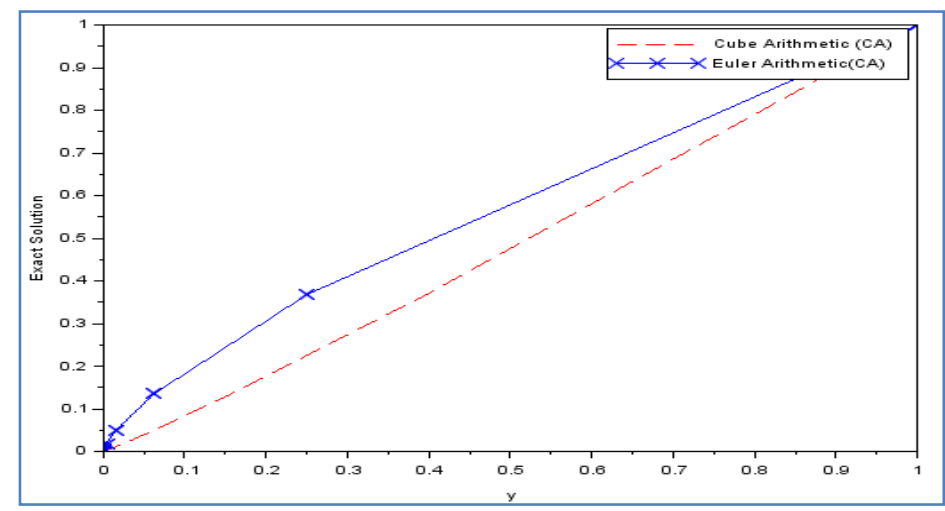

Figure 1. Differences between the two methods

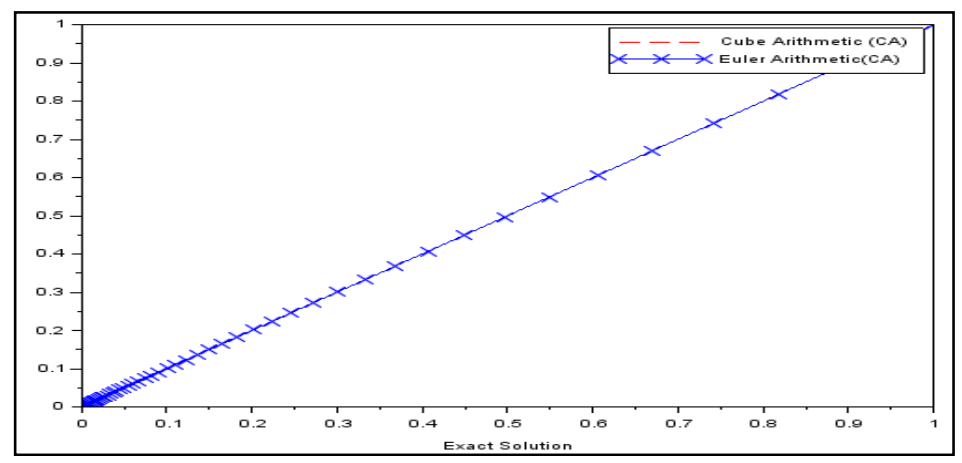

Figure 1. The results for both CA and EA methods 
Problem 3 demonstrated that the CA method scored better results compared to EA at each step size. At $h=0.1$, CA scored 0.029020 whilst EA scored 0.117900 , and at $h=0.01$, CA scored 0.000300 whilst EA scored 0.00040, as shown in Figure 3.

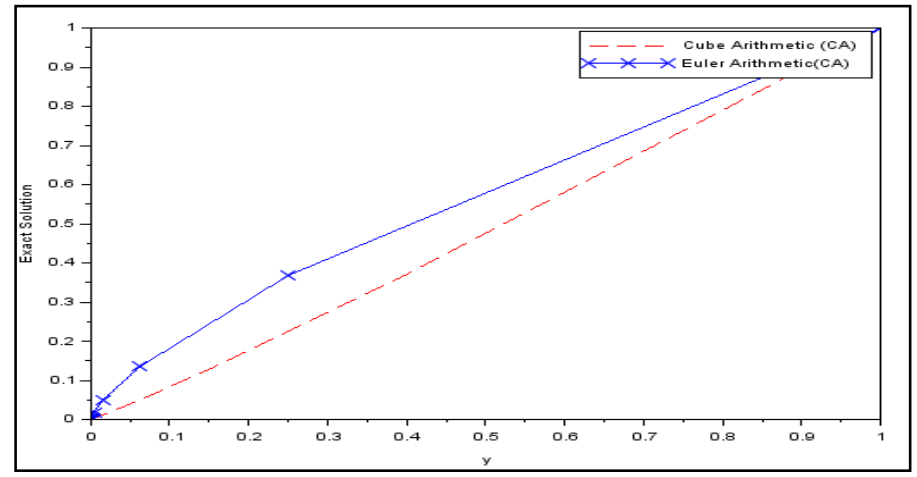

Figure 2. The results for both CA and EA methods

It can be summarized that the proposed CA method provides a more accurate result than EA in solving ODEs at higher step size.

\section{CONCLUSION}

This paper has proposed Cube Arithmetic, a new method using modified Euler as finding of this study. The feasibility of the proposed algorithm was compared with Euler Arithmetic and tested using SCILAB. Subsequently, the Cube Arithmetic compared with Euler Arithmetic scheme with the exact solution. Usually, the ordinary Euler method using a small step size gives the solution almost to the exact solution. However, in this study has been demonstrated that Cube Arithmetic provided solutions that are similar to exact solutions at small step size and also at higher step size. The benefits of using small step size will give a higher accuracy. Therefore a higher step size would reduce complexity and processing time. As a conclusion, Cube Arithmetic can be used as an alternative algorithm to solve ODE problems.

\section{ACKNOWLEDGMENTS}

The authors gratefully acknowledge the Faculty of Science and Technology Defense, Universiti Pertahanan Nasional Malaysia. The author Nooraida Samsudin also would like to thank TATI University College for the Short Term Grant (STG 1/2018).

\section{REFERENCES}

[1] A Modified ODE Solver for Autonomous Initial Value Problems Zaib-un-Nisa Memon, Sania Qureshi, Asif Ali Shaikh, Muhammad Saleem Chandio, Mathematical Theory and Modeling www.iiste.org ISSN 2224-5804 (Paper) ISSN 2225-0522 (Online) Vol.4, No.3, 2014

[2] Z. Salleh, Ordinary Differential Equations (ODE) Using Euler's Technique and Scilab Programming, Mathematical Models and Methods in Modern Science20(4) (2012), pp. 264-269

[3] A. Jabbar, F.M. Malik, Sampled-Data Backstepping Control of a Quadrotor Unmanned Aerial Vehicle. IAES International Journal of Robotics and Automation (IJRA), Vol 4 No 2, 2015; pp. 124-134.

[4] Y.Li, Breaking the Digital Video Steganography. TELKOMNIKA Indonesian Journal of Electrical Engineering, Vol 11 No 3, 2013, PP 1691-1696

[5] P. Henrici, Discrete Variable Methods in Ordinary Differential Equation, John Wiley \& Sons, 1962, pp. 187-190.

[6] E. Kreyszig, Advanced Engineering Mathematics, 9th ed., John Wiley and Sons, 2006, pp. 19-20

[7] S. Qureshi, M.S. Chandio, I.A. Junejo, A.A. Shaikh and Z.-U.N. Memon, On Error Bound for Local Truncation Error of an Explicit Iterative Algorithm in Ordinary Differential Equations,Sci.Int.(Lahore)26(3) (2014), pp. 14131415.

[8] N.M.M. Yusop and M.K. Hasan and M. Rahmat, Comparison New Algorithm Modified Euler in Ordinary Differential Equation Using Scilab Programming. Lecture Notes on Software Engineering, 2015. 3(3): pp. 199- 202 
[9] S. Fadugba, B. Ogunrinde and T. Okunlola, Euler's Method for Solving Initial Value Problems InOrdinary Differential Equations, ThePacific Journal of Science Technology 13(2)(2012), pp.152- 158.

[10] N.M.M. Yusop, M.K. Hasan, M. Wook, M.F.M. Amran, S.R. Ahmad,Comparison New Algorithm Modified Euler Based On Harmonic-Poly gon Approach For Solving Ordinary Differential Equation, Journal of Telecommunication, Electronic and Computer Engineering, Volume 9, Issue 2-11, 2017, pp. 29-32

[11] N.M.M. Yusop, M.K. Hasan, M. Wook, M.F.M. Amran, S.R. Ahmad, A new Euler scheme based on harmonicpolygon approach for solving first order ordinary differential equation. The 2nd International Conference On Applied Science And Technology 2017 (ICAST'17)

[12] F. Agus, Haviluddin, Scilab software as an alternative low-cost computing in solving the linear equations problem. AIP Conference Proceedings (2017)

[13] N.M.M. Yusop and M.K. Hasan, Development of New Harmonic Euler Using Nonstandard Finite Difference Technique for Solving Stiff Problems, Jurnal Teknologi (Sciences and Engineering), 7(22) (2014), pp. 19-24

[14] M.A.Islam, Accuracy Analysis of Numerical solutions of initial value problems (IVP) for ordinary differential equations (ODE). IOSR Journal of Mathematics. Volume 11, Issue 3 Ver. III (2015), PP 18-23

[15] INRIA-ENPC (2017). Scilab (Version 6.0). URL http://www.scilab.org/

[16] Zarina, B.I., Mohamed, S., Khairil, I. and Zanariah, M., Block Method for Generalised Multistep Adams and Backwards Differentiation Formulae In Solving First Order ODEs. MATEMATIKA, 2005. 21(1): p. 25-11

[17] R. Jaiswal, A. A. Pathan, Study of Numerical Analy sis - Differential Equation International Journal of Advanced Research in Computer Science and Software Engineering, Volume 5, Issue 10, (2015) 